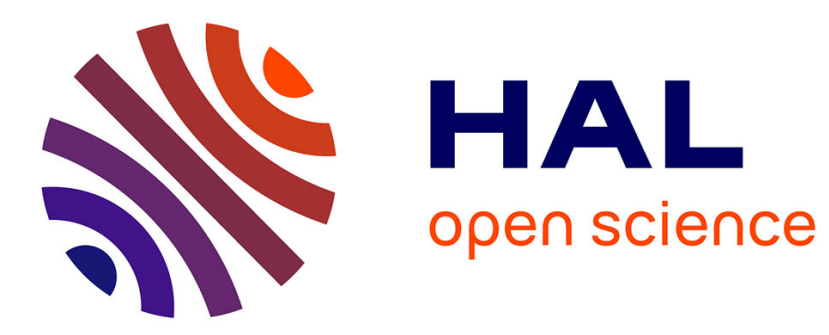

\title{
Faire la frontière dans les murs du laboratoire Mélanie Gourarier
}

\section{To cite this version:}

Mélanie Gourarier. Faire la frontière dans les murs du laboratoire: Destins migratoires et usages de l'ADN aux États-Unis. 2017. hal-01561104

\section{HAL Id: hal-01561104 https://hal.science/hal-01561104}

Preprint submitted on 8 Aug 2017

HAL is a multi-disciplinary open access archive for the deposit and dissemination of scientific research documents, whether they are published or not. The documents may come from teaching and research institutions in France or abroad, or from public or private research centers.
L'archive ouverte pluridisciplinaire HAL, est destinée au dépôt et à la diffusion de documents scientifiques de niveau recherche, publiés ou non, émanant des établissements d'enseignement et de recherche français ou étrangers, des laboratoires publics ou privés. 


\section{Faire la frontière dans les murs du laboratoire Destins migratoires et usages de l'ADN aux États-Unis}

Mélanie Gourarier

Chargée de recherche CNRS

Un homme né en 1946 à Saint-Domingue en République dominicaine pousse la porte de l'un des centres spécialisés dans le prélèvement de l'ADN ${ }^{1}$ qui émaillent la ville de New York. Il est ouvrier spécialisé dans une usine de plastique sur l'île de Manhattan et vit dans un brownstone de Harlem. Dans la poche intérieure de son veston, une enveloppe contient les huit cents dollars qui lui permettront de payer deux tests ADN, le sien et celui de sa fille. Dans l'ambassade américaine de Saint-Domingue, la même scène est en train de se jouer. Une femme de 36 ans souhaite rejoindre son père et immigrer aux États-Unis. Elle fait face à l'agent d'administration en charge d'effectuer le prélèvement à l'aide d'un coton-tige. Il sera expédié le jour même pour analyse au laboratoire de Brooklyn qui a la charge d'organiser l'opération et où s'est rendu son père. D'ici trois à huit semaines, le verdict tombera, directement adressé aux services de l'immigration des États-Unis. La comparaison de l'ADN de ces deux individus déterminera consécutivement un lien familial et un destin migratoire, tous deux adossés à une «preuve » génétique.

Ces scènes de la vie ordinaire d'un centre spécialisé dans le prélèvement de l'ADN et d'une ambassade étatsunienne apparaissent sensationnelles pour l'observateur à plusieurs égards. Elles font d'abord sensation car elles frappent les esprits par le caractère de nouveauté que recouvre le dispositif de contrôle et d'administration de la preuve. Comment comprendre le recours aux données génétiques pour vérifier et décider d'un lien entre les personnes? Qui encadre ces usages? Et quels régimes de vérité sont ainsi mis en concurrence entre les États émetteurs des actes civils et celui récepteur des demandes de regroupement familial en charge d'évaluer l'authenticité des déclarations et des papiers ? Sensationnelles, ces scènes le sont donc également parce que saturées d'inquiétudes : celle des personnes quant à leur destin migratoire et à leur histoire familiale et celle des institutions quant à l'encadrement des flux migratoires dans l'hypothèse de fausses déclarations ou de demandes frauduleuses. C'est à la résolution de ces inquiétudes que s'intéresse cet article : les tests ADN permettent-ils de faire valoir des droits à l'immigration ou génèrent-ils une suspicion systématique à l'égard de certaines personnes et de leurs pays d'origine ? Dès lors, la filiation en contexte d'immigration fait-elle l'objet d'un traitement différentiel comparativement à d'autres situations familiales nationales ?

À partir d'une ethnographie d'un centre de tests ADN mandaté par les services états-uniens de l'immigration, cet article examine la manière dont les acteurs vivent et interprètent la suspicion qui pèse à la fois sur la légitimité des projets migratoires, sur les liens familiaux et sur les États de provenance des demandeurs. En établissant ou en contrariant un lien de filiation, les résultats d'un test ADN permettent non seulement de décider des trajectoires des personnes, mais supplantent également les vérités produites par les pays d'origine lorsque les documents d'état civil ne concordent pas avec ces résultats. En se concentrant sur la pratique des professionnels et l'expérience des usagers, l'étude du dispositif d'encadrement des tests ADN permettra de saisir comment les candidats à l'immigration sont amenés à éprouver la frontière en faisant l'expérience de ce qui devient étranger à soi, à la famille et, bien au-delà, à la nation (Lemke et Rödel 2006 ; Fassin 2009). 
Dispositif ethnographique

Les résultats de l'enquête présentés ici sont le fruit d'un travail ethnographique d'une durée de quatre mois au sein d'un centre de prélèvement de l'ADN - nommé le DNA Lab - durant lequel j'ai assisté aux consultations qui précèdent et suivent l'examen génétique. Une série d'entretiens non directifs a été menée avec trente usagers à l'issue de ces consultations.

Loin de l'atmosphère aseptisée associée couramment à l'expertise génétique, les locaux du DNA Lab sont particulièrement exigus et délabrés. Les consultations qui réunissent parfois jusqu'à quatre personnes se déroulent dans une pièce de moins de dix mètres carrés dans laquelle tient l'imposant bureau du directeur (Jacob), le poste de son assistante (Toinette) ainsi qu'une chaise coincée dans le renfoncement de la porte qui m'est attribuée pour mes observations. Une telle promiscuité rend ma présence gênante pour le bon déroulement des consultations ; aussi à la demande de Jacob, je revêts la blouse blanche règlementaire, davantage pour me fondre dans le décor que par mesure d'hygiène. Associée à l'équipe médicale, il m'est aisé d'entrer en interaction avec les usagers, mais une telle confusion autour des raisons de ma présence soulève un certain nombre de questions éthiques. La première concerne la confidentialité de matériaux aussi sensibles que ceux relatifs à la génétique. N'ayant pas accès aux informations identifiantes de l'ADN - et n'ayant d'ailleurs pas les compétences pour les lire -, je considère la valeur accordée à ces données comme un élément de l'analyse. La seconde question éthique a également trait aux données sensibles, mais davantage du point de vue de l'intime. Quelle juste posture adopter lorsque l'on touche à des éléments qui ont parfois trait à des « secrets de famille »? La réponse à cette question ne peut être univoque. J'ai choisi d'y répondre en explicitant les raisons de ma présence au début de chaque consultation afin de donner la possibilité aux usagers de la refuser. Les rares moments de l'enquête lors desquels j'ai été invitée à quitter le bureau sont survenus lors de la négociation du prix des tests ADN, à la demande de Jacob comme des usagers. C'est donc la dimension économique du processus qui a fait l'effet d'une «gêne », bien plus que les significations accordées à la génétique ou les histoires personnelles et familiales. Par ailleurs, les données recueillies en phase d'observation et celles produites lors des entretiens en tête-à-tête ont été anonymisées (noms et lieux changés) afin que les récits reproduits ici ne puissent pas être attribués aux personnes que j'ai rencontrées.

\section{La filiation dans l'immigration}

Dans un article qu'ils consacrent aux transformations du rapport aux savoirs sur la génétique, Frédéric Keck et Paul Rabinow (2006) situent la fin des années 1980 comme un moment d'euphorie dans l'usage de l'ADN qui n'a jamais cessé de croître depuis. Cet engouement s'est traduit, dans les années 2000, par une diversification et une vulgarisation de ces savoirs grâce aux tests ADN. Réservés jusqu'alors à la police scientifique ainsi qu'à la médecine légale et préventive (Vailly, Kher et Niewöhner 2011), l'expertise génétique est devenue accessible au grand public à partir de 2006 à travers la création d'entreprises commercialisant des tests ADN directement sur Internet, tels que 23andMe ou Pathway Genomics ${ }^{2}$. À partir d'un prélèvement salivaire envoyé par voie postale puis examiné en laboratoire, ces entreprises proposent à leurs clients d'évaluer les prédispositions à développer certaines maladies, les origines ethniques, la généalogie ou encore certains traits comportementaux. La 


\section{Version auteure \\ Acceptée pour publication \\ A paraître dans Genèses}

pertinence scientifique de ces tests est régulièrement l'objet de controverses, notamment autour des présupposés idéologiques qui conduisent les laboratoires à réifier des caractéristiques génétiques pour mieux classifier des groupes ethniques, dès lors figés en dehors de l'histoire et des mouvements de populations (Bonniol et Darlu 2014 ; Rajagopalan et Fujimura 2012).

Ces tests font par ailleurs l'objet de régulations spécifiques concernant la propriété des données génétiques et leur utilisation (Canselier et Desmoulin-Canselier 2011). Il existe de grandes variations dans les législations régissant l'usage des tests $\mathrm{ADN}$ selon les pays, en fonction des lois de bioéthique ainsi que des contextes politiques et économiques nationaux. En France par exemple, il est interdit de vendre ou d'avoir recours à une analyse d'ADN en l'absence d'une prescription médicale ou d'une décision de justice. La plupart des usagers ont alors recours aux services d'entreprises situées à l'étranger dont certaines s'adressent explicitement à une clientèle française, principalement par le biais de leur site Internet - ainsi le site britannique du DNA Diagnostic Center, dont le nom de domaine « testdepaternite. $\mathrm{fr}^{3}{ }^{»}$ est révélateur. Aux États-Unis, les tests sont en revanche aisément accessibles et légaux. Toute personne majeure peut se rendre dans un centre de prélèvement, commander un test $\mathrm{ADN}$ sur Internet ou en acheter un dans une pharmacie sans restriction, mis à part dans l'État de New York qui requiert une ordonnance médicale. La firme leader sur le marché états-unien estime ainsi en 2015 le nombre de ses usagers à plus d'un million alors qu'elle n'en comptait «que » 100000 en $2011^{4}$. Dans ce contexte, l'expansion des usages de la génétique se traduit autant par une croissance du nombre des usagers que par une diversification des champs d'application.

Si cette dynamique soulève de nombreux enjeux discutés et pris en charge à travers des politiques de régulation (légales, éthiques, économiques), elle suscite également l'intérêt des sciences sociales. Au-delà de leur caractère de nouveauté, les multiples utilisations des tests ADN s'inscrivent dans la généalogie des techniques de contrôle de «l'état » des personnes (Rose 2007 ; Makaremi 2007). Le terme d'état est utilisé ici tant dans son acception juridique ${ }^{5}$, médicale, morale que politique. Des études ont ainsi porté sur la place de l'ADN dans les procédures de recherche et de détermination de paternité (Fonseca 2002 ; Martial 2008). D’autres travaux s'intéressent aux usages de l'ADN pour déterminer un état de santé (Vailly, Kher et Niewöhner 2011 ; Ducourneau et Beaudevin 2011), identifier un corps (Gandsman 2009), confondre un coupable (Juston 2016), ou encore chercher des origines familiales (Legrand 2009) ou ethniques (Wade 2009 ; Nelson 2016). Tous soulignent que l'examen de l'ADN est mobilisé, d'une façon ou d'une autre, pour résoudre une incertitude relative à la détermination des personnes. Dès lors, par leur qualité d'innovation, les tests ADN transforment-ils radicalement les procédures de regroupement familial ? En d'autres termes, le recours à l'examen génétique pour déterminer des filiations génère-t-il de nouvelles pratiques d'authentification des demandes ou s'inscrit-il davantage dans une généalogie administrative plus longue de soupçon à l'égard des migrants ?

Comprendre si la filiation en contexte d'immigration fait l'objet de traitements spécifiques aux États-Unis nécessite de s'intéresser plus largement aux transformations des conceptions de la parenté à l'épreuve des nouvelles technologies de la reproduction. Ces biotechnologies opèrent-elle une dissociation entre la reproduction biologique et la reproduction sociale, ou conduisent-elles à renforcer le lien entre les deux (Weber 2013 : 6) ? Ce questionnement a marqué le renouveau de l'anthropologie de la parenté depuis la critique adressée à la discipline par David Schneider (1984) et les études consacrées aux nouvelles formes de parenté (Strathern 1992 ; Porquerès i Gené 2009 ; Courduriès et Herbrand 2014). Si l'ambition de cet article n'est pas de résoudre 


\section{Version auteure \\ Acceptée pour publication \\ A paraître dans Genèses}

l'opposition conçue comme aporétique entre conception biologique et conception sociale de la parenté ${ }^{6}$, il vise à montrer l'intérêt de situer ces mises en opposition afin de mieux en souligner les enjeux sociaux. C'est notamment la perspective adoptée par Florence Weber dans son ouvrage consacré à l'articulation du « sang » (biogénétique), du «nom » (juridique) et du « quotidien » (pratique) dans les représentations ordinaires de la filiation (Weber 2013). Plus que leur opposition, c'est la prévalence d'une logique sur une autre que l'auteure examine à partir de cas ethnographiques où parenté biogénétique, parenté légale et parenté quotidienne se trouvent dissociées. L'usage des tests ADN dans certaines procédures de regroupement familial permet alors d'éclairer ces questionnements. Alors qu'il existe de par le monde de multiples manières de « faire famille »notamment par l'adoption mais aussi, dans certains pays, par la gestation pour autrui ou le mariage entre personnes de même sexe (Courduriès et Herbrand 2014) -, le recours aux tests ADN suppose que la seule filiation légitime et recevable, en situation d'immigration, est celle des « liens du sang » établie par l'administration états-unienne, et non pas celle du quotidien déclarée par les requérants, ni celle du droit émise par les États d'origine. L'accent porté sur la parenté génétique, lorsque celle-ci est discordante vis-à-vis de la parenté légale et/ou quotidienne versée aux dossiers, présente donc des effets d'imposition : la "vérité » établie par les services de l'immigration supplante celle déclarée par les personnes et attestée par les documents d'état civil. Or, la force de cette imposition repose sur une logique du soupçon à l'égard des demandeurs qui s'inscrit dans une histoire de plus longue durée.

Loin de découler de techniques de contrôle nouvelles que permettent notamment les tests $\mathrm{ADN}^{7}$, le soupçon qui pèse sur les migrants a été analysé comme relevant d'un processus long de criminalisation et de racialisation de ceux-ci (Ceyhan et Tsoukala 2002 ; Pallida 1999). Ce processus s'inscrit dans une dynamique internationale quand ceux qui migrent sont « définis comme des "clandestins" qui menaceraient de pénétrer le territoire » (Pallida 1999 : 94) - et nationale, notamment par l'intensification des pratiques de contrôle dont font l'objet les immigrés légaux. Dans un article qu'elle consacre aux propositions de « lois Sarkozy » portant réforme des lois sur l'immigration, Chowra Makaremi (2007) s'interroge sur les raisons des crispations politiques au sujet d'étrangers demeurant légalement sur le territoire, alors que l'immigration, dans le cadre de regroupements familiaux, représente une portion congrue des entrées légales (11\%). Elle s'intéresse plus particulièrement au déplacement de la criminalisation des migrants vers des suspicions relatives aux unions et aux réunions familiales. Selon elle, «alors que des figures comme celles du clandestin et du faux demandeur d'asile prennent de l'ampleur, le soupçon et l'illégitimité se transposent sur les étrangers vivant légalement dans les pays européens : luttes contre les mariages blancs, durcissement du regroupement familial »(ibid.). En France, cette dynamique est devenue particulièrement saillante lors des discussions autour de la loi Hortefeux de 2007 dont l'une des propositions concernait justement l'usage des tests ADN dans les procédures de regroupement familial. Or, cette transposition du soupçon d'une immigration illégale que dissimuleraient des alliances et des filiations illégitimes s'opère également du demandeur vers sa nation d'origine. L'authentification des liens par la comparaison des gènes de deux individus conduit ainsi à affirmer non seulement une vérité nouvelle sur l'histoire familiale, mais aussi un régime de preuve supérieure à celle produite par les États émetteurs des papiers. L'auteur de l'amendement sur les tests ADN, Thierry Mariani, ne dit pas autre chose lorsque, pour défendre son projet, il affirme que « les tests $\mathrm{ADN}$ c'est uniquement dans les pays où l'état civil est douteux, ou bien où il y a une absence d'état civil. Je rappelle quand même qu'aujourd'hui, on a des pays où l'état civil a 


\section{Version auteure \\ Acceptée pour publication \\ A paraître dans Genèses}

disparu $^{8} ! »$ Au-delà des accusations de fausses déclarations des requérants, ce sont bien ici les États des pays de provenance des migrants qui deviennent l'objet principal des suspicions.

En France, la proposition d'inclure le recours aux tests ADN dans les procédures de regroupement familial n'a, à ce jour, pas été mise en application'. Ce projet d'amendement a généré de très vives oppositions dans le débat public, politique et législatif. Le Comité consultatif national d'éthique (CCNE) pour les sciences de la vie et de la santé a notamment exprimé les réserves suivantes dans un rapport commandité par l'Assemblée nationale :

«D'une manière générale le CCNE attire l'attention sur la dimension profondément symbolique dans la société de toute mesure qui demande à la vérité biologique d'être l'ultime arbitre dans des questions qui touchent à l'identité sociale et culturelle. Elle conduirait furtivement à généraliser de telles identifications génétiques, qui pourraient se révéler à terme attentatoires aux libertés individuelles. Elle risquerait d'inscrire dans l'univers culturel et social la banalisation de l'identification génétique avec ses risques afférents de discrimination.

Le CCNE redoute les modalités concrètes d'application dans des réalités culturelles très différentes des nôtres. Nos concitoyens comprendraient peut-être mieux l'exacte réalité de tels enjeux s'ils étaient confrontés à des exigences analogues lors de leur propre demande de visa ${ }^{10}$.»

Ce qui est incriminé ici n'est pas tant la valeur accordée à l'ADN en tant que telle que le traitement différentiel qui émergerait de facto entre les immigrés légaux et les citoyens nationaux (Lemke et Rödel 2011 ; Ilpo 2014). Au final, l'option des tests ADN en matière de regroupement familial a donc été récusée en France au nom de trois arguments complémentaires : d'abord le refus de réduire la complexité vécue des liens de filiation à la seule biogénétique, ensuite celui de discriminer les migrants vis-à-vis des nationaux du point de vue des formes possibles de «faire famille», enfin celui du droit à l'inaliénabilité des données génétiques.

Aux États-Unis, à ma connaissance, de tels débats n'ont pas eu lieu. Les usages de l'ADN dans les procédures de regroupement familial semblent être passés dans les pratiques bureaucratiques depuis 2005 sans que cela n'ait généré les mêmes résistances qu'en France. L'analyse du recours aux tests ADN dans ce contexte permet donc de saisir concrètement les enjeux évoqués par le CCNE : que signifie l'identification génétique lorsqu'elle ne s'applique qu'aux immigrants dans l'exercice de leur droit au regroupement familial ?

\section{Situer l'ADN : enquêter à Brooklyn}

Le centre spécialisé dans le prélèvement de l'ADN où j'ai enquêté se situe dans un dispensaire médical du sud de Brooklyn qui propose des soins gratuits. Le DNA Lab y loue des locaux moyennant un loyer modeste ${ }^{11}$. En raison de sa localisation dans un quartier historiquement afro-américain, caribéen et hispanique, encore préservé de la gentrification, les problématiques rencontrées par le DNA Lab se différencient nettement de celles des autres centres établis à Manhattan, où la clientèle visée correspond davantage aux classes moyennes et supérieures qui y résident. C'est ce que souligne Jacob, son directeur, lors d'un entretien d'octobre 2015 :

«En m'installant ici, c'est certain que je visais une population différente, très spéciale, avec une problématique sociale très claire. Mais c'est aussi ça qui me plaisait, me frotter aux problèmes des communities. Si j'avais voulu avoir la paix et m'occuper de gens qu'ont pas d'ennuis à part se demander si leur femme les trompe, je me serais installé dans l'Upper East Side $^{12}$ ! » (Entretien, octobre 2015)

Dans la pratique d'un centre de prélèvement de l'ADN, « se frotter aux problèmes des communities » signifie se spécialiser dans un certain type de test. Lors de ses débuts à Brooklyn il y a une quinzaine d'années, Jacob travaillait surtout avec des particuliers souhaitant réaliser des tests de paternité à des fins privées. Depuis deux ans en revanche, il s'est spécialisé dans les « tests d'immigration » (immigration tests) afin de répondre à la demande croissante des services de l'immigration qui lui adressent des candidats au regroupement familial. Il 


\section{Version auteure Acceptée pour publication A paraître dans Genèses}

existe en effet une liste produite par le Département d'État (Department of State) recensant, dans chaque État, les centres de prélèvement et les laboratoires accrédités. L'administration adresse systématiquement cette liste aux requérants dès lors qu'un test ADN est envisagé ${ }^{13}$. Pour figurer dans cette liste, Jacob a déposé une demande spécifique aux services de l'immigration de l'État de New-York qui ont validé son dossier après vérification de ses diplômes et des conditions de l'exercice de sa profession (locaux, personnels). Aujourd'hui, les tests d'immigration constituent selon lui près de $70 \%$ des tests réalisés au sein de son laboratoire. Les $30 \%$ restants sont des tests dits de «curiosité », appelés peace of mind, et des tests de paternité dits « légaux » (legal paternity test). Les premiers ne nécessitent pas le consentement de l'ensemble des parties et n'ont pas de valeur juridique tandis que les seconds, plus coûteux (400 dollars par personne prélevée contre 250 dollars pour le test de curiosité), sont reconnus devant les tribunaux.

Le choix d'enquêter sur ce centre, dû initialement aux aléas de la négociation de l'accès au terrain, a donc orienté de façon conséquente ma recherche. Axées au départ sur des questions de parenté, mes préoccupations se sont peu à peu déplacées vers des questions migratoires, en lien avec la détermination des filiations. Sur les quatre mois de l'enquête conduite au DNA Lab, les usagers rencontrés étaient à plus de 90 \% Noirs de migration récente (République dominicaine, Jamaïque, Haïti ou encore Afrique francophone, notamment Sénégal, Cameroun ou Mali), les 10 \% restants ayant pour l'essentiel émigré du Costa Rica, du Mexique, de l'Inde ou du Pakistan. Je n'ai rencontré que deux familles blanches - l'une de Russie et l'autre de l'État américain du Dakotadu-Sud -, toutes deux venues au DNA Lab pour réaliser uniquement des tests de curiosité. La quasi-totalité des personnes rencontrées vivait dans les quartiers pauvres de Brooklyn, de Harlem ou du New Jersey et exerçait des métiers peu qualifiés et faiblement rémunérés (agents de la fonction publique, ouvriers non qualifiés, etc.). Par ailleurs, les deux seules personnes qui travaillent au DNA Lab sont également issues des minorités. Le directeur Jacob, docteur en pharmacie, est un Français juif orthodoxe de 47 ans immigré aux États-Unis depuis le milieu des années 1980. Il vit à Marine Park, un quartier résidentiel de la classe moyenne situé dans le sud de Brooklyn avec ses sept enfants et sa femme d'origine israélienne. Toinette, sa jeune assistante de 26 ans, est née en Jamaïque. À la mort de sa mère quand elle avait 7 ans, elle a été envoyée chez son père à Chicago. À présent elle vit à Bed-Stuy, un quartier afro-américain du centre de Brooklyn, avec son mari (un Américain d'origine jamaïcaine) et leur fils de 6 ans.

L'ethnographie de ce laboratoire conduit à s'intéresser à la dimension située des pratiques de l'ADN, non pas seulement du point de vue des usagers, mais aussi des encadrants. Jacob impute ainsi ce qu'il envisage comme un respect des valeurs morales des effets sur sa pratique professionnelle. Aussi majore-t-il ou minimise-t-il le poids de l'ADN en fonction des risques de ruptures familiales produites au moment de l'annonce d'un résultat :

«-C'est certain que pour moi, c'est important aussi de faire en sorte qu'il n'y ait pas de drame si un test n'est pas bon. Parce que bon, je suis un scientifique, mais je suis aussi juif. Donc pour moi, [...] la famille c'est une valeur importante [...]. Quand on dirige un laboratoire comme moi, il faut savoir qu'on a un certain pouvoir. Avec ma position je peux faire attention à pas briser des familles. Si je peux éviter un avortement ou un divorce, c'est certain que c'est aussi pour moi une question d'éthique. [...]

- Et comment tu t'y prends?

- Je vais te dire, ça, ça dépend vraiment des cas. Chaque situation est différente. Tu peux orienter aussi le résultat en expliquant bien aux clients. Si tu vois qu'un homme est attaché à son enfant et que le test revient pas bon, tu insistes avec lui sur ce point pour pas qu'il y ait de rupture du lien.

- C'est-à-dire, par exemple ? 


\section{Version auteure \\ Acceptée pour publication \\ A paraître dans Genèses}

- Moi ce que je fais dans ce cas-là, c'est que j'explique que la génétique c'est une chose, mais que l'amour ça peut aussi être autre chose et que parfois c'est plus important. » (Entretien, octobre 2015, Brooklyn NY)

La possibilité de relativiser la force de l'ADN en fonction des situations sociales et familiales des personnes concernées est présentée ici comme une moralisation de la pratique scientifique conciliée à l'éthique. Cet extrait d'entretien montre que les significations accordées à la génétique ne sont pas univoques. Elles ne dépendent pas seulement de ceux qui les formulent, mais varient aussi en fonction des situations dans lesquelles elles s'inscrivent. L'analyse de l'ADN est donc travaillée et transformée en permanence par les acteurs, qu'ils la façonnent dans leur pratique professionnelle quotidienne ou qu'ils soient contraints de se livrer à un test par les services de l'immigration. Or, ces arrangements avec les savoirs scientifiques et législatifs ne ressortissent pas seulement de l'ordinaire des pratiques : ils témoignent aussi de la spécificité accordée aux conceptions de la filiation en contexte migratoire.

\section{Généalogie d'une suspicion : l'administration des tests ADN aux États-Unis}

Loin d'être requise de façon systématique, la réalisation d'un test $\mathrm{ADN}$ dans le cadre des procédures de regroupement familial dépend largement de l'appréciation du fonctionnaire en charge du dossier. Si l'enquête menée dans un centre de prélèvement de l'ADN ne permet pas d'évaluer les modalités de cette appréciation en l'état actuel de la recherche ${ }^{14}$, on peut néanmoins saisir la variabilité des pratiques administratives par le biais des dossiers qui parviennent au DNA Lab. Ces cas permettent d'approcher, à partir du récit des acteurs, leurs expériences de la bureaucratie. En outre, l'attention portée ici aux experts de la génétique conduit à interroger le poids des pratiques professionnelles dans l'imposition d'une « vérité » sur les liens de filiation.

À la recherche de documents administratifs et surtout d'un texte de loi sur l'usage des tests ADN dans les procédures de regroupement familial, j'ai interrogé la responsable d'un programme d'aide juridique aux migrants au sein d'une association catholique à but non lucratif située de New York, à propos du cadre législatif états-unien. Elle m'a alors expliqué, non sans ironie, la raison de mes recherches infructueuses :

«- Il n'y a pas de texte de loi contre..., ça veut pas dire qu'il y en a un pour ! Vous comprenez ? C'est possible puisque rien ne l'interdit. C'est comme ça que ça fonctionne ici [aux États-Unis]. Alors vous pouvez toujours perdre votre énergie à chercher la loi, à ma connaissance elle n'existe pas [...]. Mais surtout je ne comprends pas le sens de votre question. Que voulez-vous savoir exactement? C'est simplement une possibilité offerte aux requérants.

- C'est-à-dire que c'est interdit en France. Il y a eu beaucoup de discussions en 2007. Un projet de loi devait passer sur la possibilité d'effectuer un test ADN pour vérifier des liens de filiation. La loi n'est pas passée car ça a suscité un débat public avec des tribunes, des prises de position dans les médias. Du coup j'aimerais savoir comment cela s'est passé aux États-Unis. S'il y a eu des discussions de ce type.

- Mais c'est interdit en France?

- Oui.

- Vraiment ?

- Oui.

- Mais, je ne comprends pas. Pourquoi vous n'utilisez pas l'ADN!

- Principalement pour des raisons éthiques...

- Quoi ? Ce n'est pas croyable!» (Entretie, octobre 2016, Manhattan, NYC)

L'explicitation de ce hiatus sur la valeur accordée à l'ADN de part et d'autre de l'Atlantique produit un renversement de l'étonnement ethnographique (Raulin et Rogers 2012). Alors que je cherchais des textes de loi 


\section{Version auteure \\ Acceptée pour publication \\ A paraître dans Genèses}

et d'autres traces d'un débat public concernant l'usage de la génétique dans les politiques d'immigration aux États-Unis, mon interlocutrice était déconcertée à l'idée que la France n'ait pas recours à ces techniques. Dans la mesure où, dans la majeure partie des cas, les résultats concordent avec les documents d'état civil versés aux dossiers, c'est précisément la systématisation du recours aux tests $\mathrm{ADN}$ qui me semble révéler une logique structurelle de soupçon. Or cette logique du soupçon prend, dans le discours des professionnels, la forme d'une disposition bénéfique aux usagers. Pour cette responsable associative qui accompagne et défend les migrants dans leurs démarches administratives et juridiques, l'efficacité, la véracité et la légitimité de ces tests sont indiscutables : elle les présentent sinon comme un droit, du moins comme une possibilité précieuse offerte aux requérants pour appuyer leur demande de regroupement familial. Cette façon de fonder la légitimité de l'expertise génétique en la présentant comme un recours au service des demandeurs imprègne également le discours de Jacob lorsqu'il m'interroge sur la spécificité de la législation française :

« Tu me dis que c'est interdit en France ? Vraiment je crois que c'est une erreur. Je ne comprends pas comment vous faites alors pour savoir qui est le père. Je trouve que c'est aussi une négation des droits de l'Homme. Tu comprends, ici ils ont la possibilité d'avoir ce recours. C'est un droit ! Mais bon, tu vas rentrer en France et tu vas leur dire, hein ? Tu vas leur expliquer que c'est la méthode la plus fiable ! Moi ça m'intéresse d'étendre les affaires en France. » (Entretien, novembre 2015, Brooklyn NYC)

Français installé à New York depuis vingt ans, Jacob déplore ici les restrictions imposées par le cadre législatif de son pays d'origine qu'il oppose au modèle économique libéral dont il fait la promotion et qui lui permet, en l'occurrence, de bénéficier d'un marché particulièrement lucratif et en pleine expansion (Ducourneau et Beaudevin 2011). À plusieurs reprises, il m'a d'ailleurs indiqué l'importance capitale que revêtait l'obtention de son accréditation auprès du Département d’État. Figurer sur la liste des centres de prélèvement que les services d'immigration distribuent aux candidats au regroupement familial lui assure en effet une clientèle croissante ${ }^{15}$ et constitue à ses yeux une responsabilité vis-à-vis du ministère auprès duquel il s'engage. Les tests d'immigration sont ainsi traités de façon prioritaire par rapport aux autres tests effectués dans son centre, qu'il s'agisse de la prise de rendez-vous, de l'envoi des prélèvements ou de la remise de résultats. Un tel enjeu économique - dans la mesure où les immigration tests constituent plus de la moitié du chiffre d'affaires actuel du DNA Lab - n'est pas sans effet sur la manière dont Jacob défend l'intérêt de ce type de test. Mais au-delà des considérations économiques, l'argument d'une « mise à disposition » des tests ADN en faveur des familles qui souhaitent se regrouper peut aussi être appréhendé comme une stratégie de justification morale ou humanitaire. On retrouvait d'ailleurs une logique similaire dans la proposition d'amendement de 2007 du Code de l'entrée et du séjour des étrangers et du droit d'asile en France :

«Le demandeur d'un visa pour un séjour d'une durée supérieure à trois mois [...] peut, en cas d'inexistence de l'acte civil ou lorsqu'il a été informé par les agents diplomatiques ou consulaires de l'existence d'un doute sérieux sur l'authenticité de celui-ci [...] demander que l'identification du demandeur de visa par ses empreintes génétiques soit recherchée afin d'apporter un élément de preuve d'une filiation $[\ldots]^{16}$ »

Dans un mémorandum daté de 2008 et destiné aux agents des services de l'immigration des États-Unis, il est certes précisé que « les tests $\mathrm{ADN}$ sont nécessairement volontaires [...]. Les agents sont alors autorisés à suggérer une analyse génétique quand d'autres formes de preuves se sont avérées non concluantes ${ }^{17} \gg$. Mais si les tests génétiques sont présentés, sinon comme une possibilité offerte, du moins comme un droit auquel peuvent recourir les demandeurs au regroupement familial, la mise en place d'un tel recours interroge. En effet, proposer d'apporter la preuve d'une filiation sur la base d'une authentification d'un lien biologique, c'est déjà 
constituer les demandes comme nécessairement soumises à la suspicion (Fassin et al. 2013). C'est ensuite transformer les techniques d'imposition du contrôle pour les faire reposer sur la « docilité » des requérants qui auraient le choix de montrer leur « bonne volonté » à dire et faire la « vérité » (Dubois 2003 ; Fassin et Mazouz 2007 ; Spire 2008). Or, dans la pratique, les demandeurs peuvent-ils choisir? Autrement dit, les tests ADN leur sont-ils présentés comme une option éventuelle au sein d'une procédure administrative, ou leur sont-ils imposés sans plus d'explication ${ }^{18}$ ?

L'enquête conduite au sein du DNA Lab révèle qu'il existe un traitement différentiel des demandes de regroupement familial en fonction du pays d'origine du requérant : les tests $\mathrm{ADN}$ sont quasiment systématiques pour les demandeurs originaires d'Afrique, des Caraïbes, d'Amérique latine, du Moyen-Orient et d'Asie du sud, et ce en dépit du versement d'actes de naissance aux dossiers de regroupement familial. Cette adresse systématique questionne d'autant plus qu'il n'existe pas de liste officielle produite par les services états-uniens de l'immigration répertoriant les pays dont les documents d'état civil seraient perçus comme suspects et ne suffiraient pas à faire la preuve des liens de filiation. Une circulaire mise en ligne en 2009 par le Bureau de la population, des réfugiés et des migrations (Bureau of Population, Refugees, and Migration) du Département d'État présente toutefois le recours aux tests $\mathrm{ADN}$ comme une mesure visant à répondre à des vagues de falsification des actes de naissances. Sur le site Internet du Département d'État, une page est aussi consacrée au programme contre les fraudes dans les procédures de regroupement familial. À la question « Pourquoi les ÉtatsUnis ont-ils décidé de procéder à des tests ADN pour certains candidats au regroupement familial en fonction des pays de provenance des requérants ? », la réponse est sans appel : "Cette mesure a été mise en place suite au nombre de fraudes constatées concernant les demandes provenant du Kenya avant d'être appliquée à d'autres pays d'Afrique ${ }^{19}{ }$. Le précédent kenyan aurait donc entrâné une extension de ces mesures de contrôle pour tout le continent africain, sans qu'un tel élargissement ne fasse l'objet, à ma connaissance, d'une quelconque justification de la part des services d'immigration. Cette généralisation de la suspicion pose question : sur quels critères des documents d'état civil sont-ils invalidés par l'administration états-unienne ?

Des explications d'ordre bureaucratique sont d'abord avancées par les services d'immigration et par les organisations non gouvernementales d'aide aux migrants pour défendre le bien-fondé des tests ADN. Bien souvent en effet, la lenteur des processus de regroupement familial (parfois plus d'un an) et leur coût élevé (plus de 1000 dollars par personne) découlent au moins en partie des difficultés à rassembler l'ensemble des documents d'état civil exigés, dès lors que ceux-ci peuvent avoir disparu en raison de catastrophes naturelles ou de guerres civiles - comme par exemple en Haïti, au Nigeria ou en Éthiopie. Dans ce contexte, le recours aux tests ADN est présenté comme une solution avantageuse à la fois pour les demandeurs (car plus rapide et moins coûteuse que la recherche des documents d'état civil) et pour les agents de l'administration qui se réfèrent directement aux résultats de l'analyse génétique. La perte, la disparition ou l'absence de document d'état civil ne suffit pas toutefois à expliquer ce phénomène. Certains immigrants possèdent en effet des documents produits par leurs États d'origine. Ce sont alors les États eux-mêmes qui sont suspectés par l'administration états-unienne d'une mauvaise gestion des archives biographiques et familiales de leurs ressortissants (Roux, ce volume). C'est donc bien la suspicion de fraude - relative au pays d'origine - qui préside ici à la décision d'un recours à l'expertise génétique par les services de l'immigration des États-Unis. Il n'est plus guère question d'assistance proposée aux candidats afin de faciliter leurs démarches, encore moins de droit : la réalisation d'un test ADN 


\section{Version auteure \\ Acceptée pour publication \\ A paraître dans Genèses}

relève bien plutôt ici d'une pratique d'imposition administrative. L'aide facultative censément à la disposition des migrants - selon les circulaires bureaucratiques des années 2000 - est donc supplantée dans les faits par une politique étatique de soupçon généralisé à l'égard d'autres États dont la capacité à administrer est fortement dépréciée.

\section{Éprouver la frontière}

$\mathrm{Si}$, du point de vue du Département d'État, la systématisation de l'usage des tests ADN s'explique en partie par un objectif de productivité bureaucratique (traiter un maximum de dossiers en un minimum de temps), elle reflète aussi une politique d'encadrement des flux migratoires fondée sur la suspicion. Cette dernière ne porte pas seulement sur la parole et les intentions, mais aussi sur ce que les personnes contiendraient comme "vérité », au-delà de ce qu'elles savent sur elles-mêmes. Dès lors, les tests ADN apparaissent autant comme l'outil d'une biopolitique s'inscrivant dans la continuité de pratiques de régulation du vivant (Rabinow et Rose 2006 ; Rose et Novas 2005) que comme un dispositif par lequel les individus sont amenés à éprouver la frontière. Les immigrés légaux, dans l'exercice de leur droit au regroupement familial sont sommés de faire la preuve de la légitimité de leur demande et, par là, de se ressentir, encore et toujours, comme étrangers (Sayad 1999).

Durant l'enquête conduite au sein du DNA Lab, la plupart du temps, le recours aux tests ADN semblait aller de soi et mes questions à ce sujet étonnaient les usagers au point de générer, chez eux, un certain amusement. Lorsque je demandais aux clients du centre les raisons qui les amenaient à réaliser une expertise génétique les réponses étaient souvent : «Elle est drôle votre question !», ou encore : «Je ne comprends pas pourquoi vous me demandez ça ». En les interrogeant sur les entretiens qu'ils avaient eus avec les agents des services de l'immigration, j'ai fini par comprendre qu'ils n'avaient jamais été informés de la possibilité de refuser le test $\mathrm{ADN}$. Questionné sur son expérience personnelle de l'expertise génétique, l'un des usagers du DNA Lab m'a fait cette réponse laconique : «J'en pense rien moi, je n'ai pas le choix. J'ai rien à dire. C'est le prix à payer ». La résignation de cet homme suggère que l'analyse génétique relève ici davantage d'une imposition que d'un consentement ou d'un droit des requérants. Toutefois, dans les cas - majoritaires - où les résultats se révèlent positifs $^{20}$, les usagers se montrent satisfaits de cette technique. En leur permettant d'accélérer l'examen et la validation de leur dossier, les tests ADN passent alors pour une simple formalité administrative. La force d'imposition de l'examen génétique dans la détermination des filiations dépend ainsi autant du mode d'administration des tests par les agents de l'État que de l'adhésion de ceux qui font l'objet de telles mesures.

Si les tests ADN se soldent le plus souvent de façon heureuse en validant les démarches entreprises par les demandeurs, dans certains cas en revanche, l'issue d'une recherche génétique se révèle dramatique et vient bouleverser les histoires familiales et les projets migratoires. C'est notamment le cas pour Pape, un Sénégalais d'une quarantaine d'années installé à Brooklyn depuis quinze ans. Quand je le rencontre au DNA Lab, il se réjouit de pouvoir parler en français et me raconte, sans détour, son histoire et ses craintes. Pape a d'abord été marié au Sénégal avant de divorcer peu après son installation aux États-Unis à la suite d'une mutation professionnelle dans l'entreprise d'import/export où il travaillait. Il a deux enfants de ce premier mariage, restés auprès de leur mère à Dakar et âgés de 12 et 9 ans. Il est désormais remarié avec une femme de nationalité américaine avec laquelle il a un enfant de 4 ans. En accord avec son ancienne épouse, il souhaite à présent que ses deux premiers enfants le rejoignent sur le sol américain : «Maintenant je veux qu'ils viennent. Ils sont plus grands. La maman, elle pense aussi que c'est bien pour eux. C'est bien pour leur avenir qu'ils viennent ici ». 


\section{Version auteure \\ Acceptée pour publication \\ A paraître dans Genèses}

Alors qu'il constitue le dossier de demande de regroupement familial auprès des services de l'immigration à

New York, le fonctionnaire lui fournit la liste de centres de prélèvement agréés pour réaliser un test ADN et ainsi «finaliser efficacement la demande». Pape choisit le DNA Lab pour sa proximité avec son domicile. À ses yeux, les tests ADN constituent une pièce « comme une autre » du dossier à constituer, malgré leur coût (250 dollars par personne prélevée). Dix jours plus tard, Jacob reçoit les résultats des prélèvements effectués dans son centre et à Dakar, dans l'enceinte de l'ambassade des États-Unis. Ils ne concordent pas. Je suis dans son bureau quand il décroche son combiné et compose le numéro de téléphone en m'adressant d'un air grave : «Ça va être douloureux cette fois. Les deux tests sont négatifs ». Il me fait signe d'écouter et allume le haut-parleur ${ }^{21}$ :

$\ll-[\ldots]$ Je viens juste de recevoir vos résultats. Nous avons donc deux résultats pour vous. Test négatif avec le premier enfant et test négatif avec le second

- Comment ça ? Comment ça se passe?

- Comment ça se passe... Il semblerait que ce ne soit pas vos enfants.

- Mais si ce sont mes enfants, je les ai élevés. Bien sûr que ce sont les miens, comment vous pouvez dire ça, ce n'est pas vrai ! C'est impossible, impossible vous comprenez, ce que vous me dites là ce n'est pas possible.

- Mais les tests sont revenus négatifs. Vous comprenez ? L'ADN ne correspond pas. Il faut que vous parliez avec la maman.

- Elle sait que ce sont mes enfants, qu'ils viennent de moi. Elle sait que je suis leur père. Elle ne ment pas !

- Si, elle peut mentir.

- C'est vous qui dites ça. Moi je dis qu'elle ne ment pas.

- Je comprends bien mais ce sont les résultats que nous avons.

- Je suis étonné, très très choqué. Vraiment je ne comprends pas, les bras m'en tombent, là. Le ciel me tombe sur la tête. Je ne comprends pas ce que vous dites.

- Nous étions étonnés aussi.

- Mais quelles sont les chances que vous vous trompiez?

- Zéro.

- Mais vous vous rendez compte ? Mes enfants, ils ne vont pas pouvoir venir.

- Je sais bien monsieur, je comprends bien. Mais ce ne sont pas vos enfants ${ }^{22}$. » (Entretien, octobre 2015, Brooklyn NYC)

L'issue de cette triple analyse de l'ADN (celle des deux enfants et du demandeur) s'avère dramatique en ce qu'elle scelle des destins migratoires et transforme, par « l'effet de vérité » et l'impossibilité des retrouvailles, des liens familiaux. Contrairement à la position « éthique » qu'il défend en faveur des unions familiales hors contexte migratoire, Jacob ne relativise ici à aucun moment le poids de la génétique lorsqu'il annonce un résultat. Il le renforce même en répétant à plusieurs reprises : «ce ne sont pas vos enfants ». Durant mon séjour de terrain au sein du DNA Lab, j'ai pu observer que ce mode de restitution des résultats était spécifique aux tests d'immigration pour lesquels « il n’y a rien à dire » et rien à apprécier au-delà du taux de probabilité de liens de filiation indiqué. À l'inverse, lorsqu'il s'agit de tests de paternité effectués en dehors des procédures de regroupement familial, Jacob s'est montré davantage enclin à minimiser la prégnance du lien génétique. C'est particulièrement vrai pour les tests prénataux (prenatal tests) effectués sur des femmes enceintes s'interrogeant sur l'opportunité d'une interruption de grossesse au cas où le conjoint déclaré se révèle ne pas être le géniteur. 


\section{Version auteure \\ Acceptée pour publication \\ $A$ paraître dans Genèses}

Dans ce type de situation, Jacob s'accorde un temps de discussion avec ses clientes afin de les convaincre que la « ressemblance » avec le père n'est pas une donnée de départ mais se construit : «Elles ont peur que le bébé ne ressemble pas au père. Mais moi ce que je leur dis, et ça on le voit tous les jours ici, c'est que la ressemblance ça dépend aussi de la manière dont on va élever l'enfant ». Ainsi, lorsqu'une jeune femme se présente au laboratoire à l'issue d'un test prénatal, alors que les résultats indiquent que le géniteur n'est pas l'époux, Jacob n'entoure pas au feutre jaune, contrairement à son habitude pour les tests d'immigration, le pourcentage faible qui rend impossible la filiation « légitime ». Comment expliquer une telle différence dans le poids accordé aux données génétiques qui, dans un cas, sont présentées comme indiscutables, et dans l'autre, sont justement discutées ? Et comment apprécier la relativité du rapport à l'éthique scientifique et morale de Jacob ? La « responsabilité » vis-à-vis de l'État, lorsqu'il encadre un test d'immigration, vient-elle ici supplanter l' « intérêt des familles » qu'il défend dans d'autres situations ? La valeur accordée tantôt à la génétique, tantôt à la pratique et au quotidien, dépend bien ici des personnes visées par les résultats d'un test ADN, qu'il s'agisse de citoyens nationaux ou d'immigrés suspectés de mentir délibérément ou par ignorance à l'administration. Bien que Jacob ait fait lui-même l'expérience de l'immigration aux États-Unis, il ne s'identifie jamais aux trajectoires et aux difficultés de ses clients. Il s'en distancie plutôt en se présentant comme un « agent de l'État » en charge des « preuves » scientifiques. L'administration de ces tests par Jacob relève dès lors d'une pratique bureaucratique qui prend les aspects d'un traitement systématique et « objectif » des faits scientifiques : seule compte la production de résultats qui ont valeur de «vérité » sur les situations familiales.

Ainsi qu'en témoigne l'expérience malheureuse de Pape, la vérification de la validité des demandes de regroupement familial produit des individus appréhendés comme nécessairement suspects. La parole du demandeur est ici radicalement mise en doute, soit qu'il ignore la « vérité » (dans ce cas c'est la mère qui est suspectée de «tromperies »), soit qu'il la subvertisse délibérément (dans ce cas c'est la requête elle-même qui est suspectée de fraude). Dans tous les cas, l'ADN supplante les autres régimes de preuve ; l'histoire et le quotidien des liens n'ont plus guère de valeur, pas plus que la parole des personnes. C'est donc ici dans l'invisible des « corps génétiques » que la preuve se révèle au moyen de tests ADN, parfois aux dépens des personnes. C'est ce que montre un second cas dont l'issue pose non plus seulement la question de l' «entrée » mais également celle du retour.

En novembre 2015, je suis sur le terrain depuis deux mois et j'ai déjà intégré une certaine routine au sein du DNA Lab. Chaque jour, j'arrive à $9 \mathrm{~h}$ dans le bureau qui sert également de salle de consultation et de prélèvement, je salue Toinette qui s'affaire déjà au téléphone, je mets ma blouse blanche et sors mon carnet en attendant Jacob afin qu'il m'explique le déroulement de la journée et m'informe, non sans une certaine excitation, des « cas intéressants » à venir. Mais ce jour-là, je n'ai pas le temps d'arriver jusqu'au bureau. Alertée de mon entrée au son de mes pas, Toinette se précipite vers moi et me retient dans le couloir. Elle est visiblement très émue. Le matin même, elle a reçu le résultat des tests ADN de Michelle, une jeune femme de 35 ans qu'elle a prise en sympathie. Comme elle, Michelle est Jamaïcaine, vit aux États-Unis depuis une dizaine d'année et a un petit garçon âgé de 6 ans. Comme elle, elle a reçu une formation d'infirmière et tente de s'occuper à distance d'un père diabétique. «Je vais m'arranger pour que tu puisses lui parler », me dit Toinette. « Elle sera là dans l'après-midi. Je l'ai appelée ce matin pour les résultats, elle est effondrée. Si tu peux lui parler, c'est bien. C'est vraiment injuste ce qui lui arrive. Je suis dégoûtée. » 


\section{Version auteure \\ Acceptée pour publication \\ A paraître dans Genèses}

J'ai rencontrée Michelle une semaine plus tôt : elle était assise dans le couloir jouxtant le bureau qui fait office de salle d'attente. À ses côtés, un homme aux cheveux grisonnants, maigre et visiblement fatigué, semblait inquiet. J'avais profité de ce temps de latence pour leur parler. Après la séparation des parents de Michelle quand elle avait une quinzaine d'années, sa mère a suivi un homme parti s'installer en Caroline du Nord où Michelle a passé son adolescence. À 20 ans, elle a rencontré son mari et s'est installée avec lui à Brooklyn. Lors de cette première discussion, je m'étais adressée à l'homme à côté d'elle. Il m'avait à peine répondu. Michelle parlait pour lui : «C'est mon père, il vient d'arriver de la Jamaïque. Il est fatigué. » Maintenant qu'elle a une situation économique stable et un appartement assez grand, Michelle souhaite que son père la rejoigne. Il y a cinq ans, ce dernier a été diagnostiqué diabétique. Avec l'âge, il a davantage besoin de soins quotidiens. Michelle est son unique enfant et il vit seul depuis son divorce. Elle se sent responsable de lui et s'inquiète de le savoir « isolé » en Jamaïque. Elle a donc entamé une procédure de regroupement familial il y a plus d'un an, au cours de laquelle les services de l'immigration lui ont indiqué que son acte de naissance n'était pas suffisant et qu'elle devait réaliser un test ADN. Le soir même, elle appelle sa mère pour l'informer de la tournure que prend sa demande. Silence. Face à la menace imminente du test génétique, la mère se sent contrainte de révéler un secret lourd de conséquences : elle doute que l'homme qui a élevé sa fille soit son géniteur. C'était il y a un an et la voix de Michelle tremble encore, ses yeux s'embrument. Son père continue : « Il a fallu du temps pour trouver l'argent du billet d'avion. Je ne voulais pas le faire [le test $A D N]$ à Kingston, je voulais le faire avec ma fille». Depuis l'aveu de la mère, un an s'est écoulé pour recueillir l'argent nécessaire ; un an que la santé du père de Michelle se dégrade ; un an que cette famille attend de savoir la « vérité » et ce qui va « leur arriver ».

Se déroulant sur un temps long, la concrétisation du recours à un test ADN de paternité produit ici une triple suspicion : celle qui plane sur la «moralité » et la sexualité de la mère, celle qui pèse sur l'authenticité et la légitimité de la demande migratoire et celle qui menace les liens familiaux. L'imbrication de ces suspicions finit par produire un état de confusion, perceptible dans les propos de Michelle lorsqu'elle se rend au centre de prélèvement pour la seconde fois afin de récupérer ses résultats.

« Je suis vraiment très choquée. Ils me disent que mon père n'est pas mon père... C'est vraiment grave je trouve, c'est très grave. Tu comprends, j'ai 35 ans et j'apprends ça ! Déjà c'est très perturbant psychologiquement, et en plus ils me traitent comme une menteuse ! [...]

- Ils? Tu parles de qui ?

- Ici, le ministère. Ils nous traitent comme des menteurs. C'est comme si on est des voyous. Les papiers ça ne suffit pas ? Ça ne suffit jamais!

- Oui, je comprends, c'est dur, c'est vraiment dur...

- Oui vraiment, je ne m'attendais pas à ça quand j'ai commencé les papiers. Je vais peut-être devoir rentrer en Jamaïque. » (Entretien, octobre 2016, Brooklyn NYC)

Toinette m'a demandé de l'attendre à la fin de sa journée. Elle a besoin de discuter, de «lâcher la pression ». C'est la première fois que je la vois aussi affectée par l'histoire d'un client du DNA Lab. Elle m'explique surtout se sentir impuissante et détester faire son travail dans ce type de situation. Elle se sent alors partie prenante d'un système qu'elle exècre parce que raciste :

«-Tu comprends, moi je suis comme Michelle finalement. Je suis née là-bas, mais je vis ici depuis des années, je me suis mariée ici, je travaille ici. Michael [son fils] est né ici [...]. Je ne me sens pas du tout Américaine. Ici c'est raciste, ça n'a pas changé en fait. Je suis très en colère, j'avais besoin de te le dire mais au bureau avec Jacob c'est difficile de parler de ça. 


\section{Version auteure \\ Acceptée pour publication \\ A paraître dans Genèses}

- Et lui qu'est-ce qu'il en pense?

- Je ne sais pas, je ne veux pas parler de ça avec lui. Bref. Je sais que pour lui ce n'est pas pareil quand même.

- C'est-à-dire ?

- Moi je suis Jamaïcaine. Ce n'est pas pareil. Je sais ce que ça fait d'être toujours traitée comme si j'étais étrangère. » (Entretien, octobre 2016, Brooklyn NYC)

Jacob, étranger aussi puisque de nationalité française et appartenant à une minorité religieuse, est d'abord ici considéré comme Blanc. Alors qu'elle désirait faire venir son père malade, Michelle s'interroge sur un retour possible en Jamaïque. Toinette, agent du prélèvement de l'ADN et donc en première ligne des effets de son analyse, se perçoit elle-même comme une étrangère. Toutes deux engagées dans un processus qui occupe une part importante de leur vie, elles savent ce que produit la suspicion. Loin de l'endosser sans colère, elles font l'expérience d'une forme de « véridiction génétique » qui les amène à demeurer à la frontière de l'État qu'elles habitent.

Au-delà des dénouements heureux ou malheureux, qu'un lien soit infirmé ou confirmé, les tests ADN, par leur caractère administrativement «obligatoire », produisent des sujets par principe suspects. Or cette suspicion ne porte pas seulement sur l'intention de fraude, le mensonge ou le parjure, mais sur ce que les corps disent audelà de l'histoire familiale et nationale des personnes. Cette substitution de «vérités » relève d'un double processus d'imposition.

Dépossédés d'une part de leur propre récit, devenu « faux », les requérants sont contraints d'accéder à leur biographie génétique. Que les requérants adhèrent à ce nouveau récit au risque de s'en trouver bouleversés ou qu'ils y résistent sans pouvoir agir sur l'issue des procédures, la « révélation » d'une analyse d'ADN conduit à éprouver la frontière dans l'expérience de la délimitation d' " un propre et [d'] un différent » (Agier $2013: 32$ ),

c'est-à-dire aussi de l'assignation à une essence individuelle extérieure à l'histoire des personnes. Les vérités personnelles se voient ainsi opposer la vérité, celle de la biologie érigée en technique de contrôle. »

D'autre part, en recomposant un passé génétique pour déterminer l'avenir des personnes, les services étatsuniens de l'immigration imposent leur propre épreuve administrative et délégitiment les vérités antérieurement établies par les États d'origine. Cette imposition est rappelée par le caractère variablement obligatoire des tests ADN suivant les États de provenance des requérants majoritairement «non Blancs ». En contraignant les candidats à authentifier leurs liens familiaux sur la base de concordances génétiques, l'administration étatsunienne applique un double standard dans l'évaluation de ce qui fait famille. Si des lois fédérales vont dans le sens d'une reconnaissance de la dimension sociale des filiations - gestation pour autrui (Courduriès, ce volume), adoption notamment par des personnes de même sexe -, les candidats au regroupement familial sont renvoyés systématiquement du côté d'une "vérité » de la génétique qui s'établit parfois aux dépens du récit administratif et narratif de leur biographie. Or c'est précisément par le biais du traitement spécifique des filiations dans l'immigration que se jouent non plus seulement des conceptions concurrentielles de ce qui fait famille, mais des inégalités dans l'administration des « citoyennetés génétiques » (Rapp, Heath et Tausig 2008 ; Rose et Novas 2005). 


\section{Ouvrages cités}

Agier, Michel. 2013. La condition cosmopolite. L'anthropologie à l'épreuve du piège identitaire. Paris, La Découverte.

Bonniol, Jean-Luc et Pierre Darlu. 2014. "L'ADN au service d'une nouvelle quête des ancêtres?», Civilisations, $\mathrm{n}^{\circ} 63: 201-219$.

Canselier, Guillaume et Sonia Desmoulin-Canselier (dir.). 2011. Les catégories ethno-raciales à l'ère des biotechnologies. Droit, sciences et médecine face à la diversité humaine. Paris, Société de législation comparée.

Ceyhan, Ayse et Anastasia Tsoukala. 2002. «The Securitization of Migration in Western Societies: Ambivalent Discourses and Policies », Alternatives, $\mathrm{n}^{\circ} 27: 21-25$.

Courduriès, Jérôme et Cathy Herbrand. 2014. «Genre, parenté et techniques de reproduction assistée : bilan », Enfances familles générations, $\mathrm{n}^{\circ} 21: \mathrm{I}$-XXVII.

Dubois, Vincent. 2003. La vie au guichet. Relation administrative et traitement de la Misère. Paris, Economica.

Ducourneau, Pascal et Claire Beaudevin. 2011. «Génétique en ligne : déterritorialisation des régulations de santé publique et formes de développement commercial », Anthropologie et santé [en ligne], $\mathrm{n}^{\circ} 3$.

Fassin, Didier et al. 2013. «Enquête de vérité. La production des décisions pour les demandes d'asile », in Didier Fassin et al., Juger, réprimer, accompagner. Essai sur la morale de l'État. Paris, Seuil : 101-135.

Fassin, Didier et Sarah Mazouz. 2007. «Qu'est-ce que devenir français? La naturalisation comme rite d'institution républicain », Revue française de sociologie, $\mathrm{n}^{\circ} 4: 723-750$.

Fassin, Éric. 2009. «Entre famille et nation : la filiation naturalisée », Droit et société, $\mathrm{n}^{\circ} 72:$ 373-382.

Fine, Agnès et Agnès Martial. 2010. «Vers une naturalisation de la filiation ? », Genèses, ${ }^{\circ} 78$ : 121-134.

Fonseca, Claudia. 2002. «Recherche de paternité et tests d'ADN. Le cas du Brésil », Cahiers du genre, $\mathrm{n}^{\circ} 32$ : 181-205.

Gandsman, Ari. 2009. «"L’appel du sang”. Le débat sur la restitution des enfants de disparus en Argentine postdictatoriale », Anthropologie et sociétés, $\mathrm{n}^{\circ} 33: 31-48$.

Ilpo, Helén. 2014. «Biological Citizenship across the Borders: Politics of DNA Profiling for Family Reunification », Distinktion: Journal of Social Theory, vol. 15, $\mathrm{n}^{0} 3: 343-360$.

Juston, Romain. 2016. "Comment une tache de sang devient-elle une preuve? Ingrédients et recettes des preuves médico-légales », Droit et société, $\mathrm{n}^{\circ} 93:$ 395-416.

Keck, Frédéric et Paul Rabinow. 2006. «Invention et mise en scène du corps génétique », in Alain Corbin (dir.), Histoire du corps, t. 3 : Les mutations du regard. Le XXe siècle. Paris, Seuil : 71-89.

Legrand, Caroline. 2009. "Génétique et recherche généalogique », in Enric Porqueres i Gené (dir.), Défis contemporains de la parenté. Paris, Éd. de l'EHESS : 133-149.

Lemke, Thomas et Malaika Rödel. 2011. «Les tests ADN dans les procédures d'immigration : les double standards de la parenté », in Janina Kehr, Jörg Niewöhner et Joëlle Vailly (dir.), De la vie biologique à la vie sociale. Approches sociologiques et anthropologiques. Paris, La Découverte : 130-158.

Martial, Agnès. 2008. "Changements de nom, changement de filiation », in Agnès Fine (dir.), États civils en questions. Papiers, identités, sentiment de soi. Paris, CTHS : 115-138.

Makaremi, Chowra. 2007. " "De l'esprit des lois". Quelques éléments de réflexion à propos de la "loi ADN" en France », Eurostudia, vol. 3, n 2. URL : http://id.erudit.org/iderudit/071838ar10.720/017839ar. 
Nelson, Alondra. 2016. The Social Life of DNA. Race, Reparations, and Reconciliation After the Genome. Boston, Beacon Press.

Palidda, Salvatore. 1999. "La criminalisation des migrants», Actes de la recherche en sciences sociales, $n^{0} 129: 39-49$.

Porquerès i Géné, Enric (dir.). 2009. Défis contemporains de la parenté. Paris, Éd. de l'EHESS.

Rabinow, Paul et Nikolas Rose. 2006. «Biopower Today », BioSocieties, $n^{\circ} 1$ : 195-217.

Rajagopalan, Ramya et Jana H. Fujimura. 2012. «Making History via DNA, making DNA from History », in Keith Wailoo, Alondra Nelson et Catherine Lee (dir.), Genetics and the Unsettled Past. The Collision of DNA, Race and History. New Brunswick (New Jersey) et Londres, Rutgers University Press : 143-163.

Rapp, Rayna, Deborah Heath et Karen Sue Taussig (dir.). 2008. «Genetic citizenship », in David Nugent et Joan Vincent (dir.), A Companion to the Anthropology of Politics. Malden, Blackwell : 152-167.

Raulin, Anne et Susan Carol Rogers (dir.). 2012. Parallaxes transatlantiques. Vers une anthropologie réciproque. Paris, CNRS Éditions.

Rose, Nikolas. 2007. The Politics of Life Itself: Biomedicine, Power and Subjectivity in the Twenty-first Century. Princeton, Princeton University Press.

Rose, Nikolas et Carlos Novas. 2005. «Biological Citizenship», in Aihwa Ong et Stephen J. Collier (dir.), Global Assemblages: Technology, Politics and Ethics as Anthropological Problems. Malden, Blackwell : 439463.

Sayad, Abdelmalek. 1999. La double absence. Des illusions de l'émigré aux souffrances de l'immigré. Paris, Seuil.

Schneider, David. 1984. A critique of the study of kinship. Ann Arbor, The University of Michigan Press.

Spire, Alexis. 2008. Accueillir ou reconduire : enquête sur les guichets de l'immigration. Paris, Liber.

Strathern, Marilyn. 1992. Reproducing the Future: Anthropology, Kinship, and the New Reproductive Technologies. New York, Routledge.

Vailly Joëlle, Janina Kehr et Jörg Niewöhner (dir.). 2011. De la vie biologique à la vie sociale. Approches sociologiques et anthropologiques. Paris, La Découverte.

Wade, Peter. 2009. «Race, identité et parenté », in Enric Porqueres i Gené (dir.), Défis contemporains de la parenté. Paris, Éd. de l'EHESS : 171-195.

Weber, Florence. 2013. Penser la parenté aujourd'hui. La force du quotidien. Paris, Éd. Rue d'Ulm.

1 Ces structures se distinguent des laboratoires d'expertise de l'ADN du fait que s'y déroulent les seules phases de prélèvement et d'annonce des résultats. L'expertise génétique est délocalisée dans un laboratoire partenaire vers lequel sont envoyés par navette journalière les échantillons.

2 URL : https://www.23andme.com; https://www.pathway.com.

3 URL : http://www.testdepaternite.fr/fr/test-de-paternite.html.

4 URL : https://mediacenter.23andme.com/blog/23andme-1million.

5 En droit privé, «l'état d'une personne est constitué par l'ensemble des règles qui définissent la personnalité juridique d'une personne physique et qui l'individualisent par rapport à sa famille et aux autres personnes. L'état d'une personne comprend principalement ses prénoms 
et nom de famille, son lieu et sa date de naissance, sa filiation, sa capacité civile, son domicile, sa situation au regard de l'institution du mariage (célibataire, marié, divorcé) » (Serge Braudo, Dictionnaire du droit privé français [en ligne], URL : http://www.dictionnairejuridique.com/definition/etat-des-personnes.php).

6 Comme l'écrivent Agnès Fine et Agnès Martial (2010:122), «l'on tend bien souvent à opposer une parenté "sociale" à une parenté "biologique", en oubliant, pour le dire simplement, que la seconde n'est pas moins "sociale" que la première ».

7 Les visas biométriques participent également de ces nouvelles techniques de contrôle des populations.

8 «Tests ADN : Thierry Mariani s'explique sur RFI », entretien radiophonique du 18 septembre 2007 sur Radio France international.

9 L'amendement a été rejeté par le Sénat le 26 septembre 2007.

10 Comité consultatif national d'éthique pour les sciences de la vie et de la santé, Avis $\mathrm{n}^{\mathrm{o}} 100$, «Migration, filiation et identification par empreintes génétiques », 10 octobre 2007, URL : http://www.ccne-ethique.fr/fr/publications/migration-filiation-et-identification-parempreintes-genetiques\#.WQbGQ8kaQfM.

11 La somme exacte ne m'a pas été communiquée par son directeur, bien qu'il justifiait son installation dans le sud de Brooklyn pour des raisons à la fois économiques (loyers moins onéreux qu'à Manhattan) et pratiques (proximité de son domicile).

12 Quartier de Manhattan dont le coût foncier est parmi le plus onéreux de New York. 13 URL :

https://travel.state.gov/content/dam/visas/SIVs/Public\%20Affiliate\%20Directory.pdf. 14 L'ethnographie d'un service de l'immigration que je projette de conduire permettra, dans le prolongement de l'enquête présentée ici, de préciser ces pratiques.

15 Cette croissance est sensible à l'échelle même de mon ethnographie dans la mesure où j'ai comptabilisé une vingtaine de tests d'immigration par semaine durant ma présence sur le terrain en automne 2015, puis trois fois plus lors de mon retour à la même période en 2016, à la veille de l'élection présidentielle.

16 Article L 111-6, URL : http://www.juritravail.com/codes/code-entree-sejour-etrangersdroit-asile/article/L111-6.html.

17 URL : https://www.uscis.gov/sites/default/files/files/pressrelease/genetic_testing.pdf. 18 Pour répondre plus concrètement à cette question, une seconde phase de l'enquête se prolongera au sein d'un service de l'immigration à New York.

19 URL : https://2001-2009.state.gov/g/prm/refadm/rls/fs/2008/112760.htm.

20 La fiabilité scientifique d'un test repose sur l'analyse statistique de petites régions de l'ADN. Ce sont plus particulièrement les allèles (expressions d'un gène) qui font l'objet d'une comparaison afin d'établir un taux de correspondance. Si celui-ci est supérieur au $99 \%$, le résultat est positif. Un rapport de filiation d'une génération peut dès lors être établi. Si celui-ci est légèrement inférieur (entre 90 et $99 \%$ ), le résultat est négatif mais permet d'établir un lien de parenté génétique entre les personnes testées. La plupart du temps il s'agit du frère, de la sœur, de la mère, du père ou encore d'un cousin ou d'une cousine du parent présumé (Bulletin de l'Académie nationale de médecine, 2009, vol. 193, $\mathrm{n}^{\circ}$ 9, 2093-2119, séance du 8 décembre 2009, URL : http://www.academie-medecine.fr/publication100036098).

21 Les résultats d'un test ADN peuvent être délivrés par téléphone si le client le souhaite. Dans tout les cas ils seront également adressés par voie postale ou délivrés en main propre dans les locaux du centre.

22 Ce dialogue a eu lieu en français. 